\title{
The CP-QAE-I: A Video Dataset for Exploring the Effect of Personality and Culture on Perceived Quality and Affect in Multimedia
}

\author{
Sharath Chandra Guntuku ${ }^{\dagger}$, Michael James Scott ${ }^{\star}$, Huan Yang ${ }^{\dagger}$, Gheorghita Ghinea ${ }^{\star}$ and Weisi Lin ${ }^{\dagger}$ \\ ${ }^{\dagger}$ School of Computer Engineering, Nanyang Technological University, Singapore \\ ${ }^{\star}$ Department of Computer Science, Brunel University, London. \\ sharathc001@e.ntu.edu.sg,michael.scott@brunel.ac.uk, hyang3@e.ntu.edu.sg, george.ghinea@brunel.ac.uk, wslin@ntu.edu.sg
}

\begin{abstract}
Perception of quality and affect are subjective, driven by a complex interplay between system and human factors. Is it, however, possible to model these factors to predict subjective perception? To pursue this question, broader collaboration is needed to sample all aspects of personality, culture, and other human factors. Thus, an appropriate dataset is needed to integrate such efforts. Here, the CP-QAE-I is proposed. This is a video dataset containing 144 video sequences based on 12 short movie clips. These vary by: frame rate; frame dimension; bit-rate; and affect. An evaluation by 76 participants drawn from the United Kingdom, Singapore, India, and China suggests adequate distinction between the video sequences in terms of perceived quality as well as positive and negative affect. Nationality also emerged as a significant predictor, supporting the rationale for further study. By sharing the dataset, this paper aims to promote work modeling human factors in multimedia perception.
\end{abstract}

\section{INTRODUCTION}

Multimedia is an active means of communication that relies on viewers' perception and an interactive process of information assimilation. For this reason, the perception of multimedia content is fundamentally subjective. Even objective multimedia system parameters (e.g. frame rate, frame dimension, bit-rate, etc.) are subjectively perceived by viewers because of their individual expectations and the degree of deviation they are willing to tolerate. This means that individual differences in human factors could moderate the impact of any particular system parameter [1]. If these human factors could be accurately modeled, then key aspects of multimedia content and delivery could be adapted to an individual in order to maximize levels of enjoyment. An application area where this would be useful could be mobile content streaming because delivery resources are often limited and mobile devices tend to be used by individuals (e.g., [2]). However, to construct such a model, the way in which human factors influence multimedia perception must be explored.

Human factors are complex because each individual is unique. Nevertheless, we each possess individual traits which vary in systematic ways and these differences can be explored. Consider personality, a series of "internal properties" that relate to overt behaviors [3]. Though there are many different theories which examine the predictive utility of personality, the Five Factor Model (FFM) [4] is one of the most parsimonious models. This consists of: openness to experience, conscientiousness, extroversion, agreeableness and neuroticism. Any of these dimensions could be mapped to perception of quality or affective experience. For example, individuals with high neuroticism might be more sensitive to multimedia content that evokes negative emotions.

Beyond personal traits, there are also traits which are associated with the culture an individual is from. These cultural traits represent "the collective programming of the mind distinguishing the members of one [nation] or category of people from others" which subsequently leads to a "broad tendency [for members of a group] to prefer certain states of affairs over others" [5]. This is because perception and cognition are informed through information that individuals sample from their local environment, which itself is shaped through shared conceptions and collective norms. Among theories of culture is Hofstede's Six Factor Model (HM) [5] which includes: power distance; individualism; uncertainty avoidance; masculinity; pragmatism; and indulgence. Again, these dimensions could interact with perception of quality or affective experience. For example, individuals with high indulgence may potentially be more sensitive to multimedia content that evokes positive emotions.

Following this line of reasoning, a model based on personal and cultural traits could be constructed to predict subjective perception. However, due to the complex nature of personality and culture, this would likely need numerous, globally distributed studies. This raises concerns about external validity: how do researchers ensure that their findings generalize to a broad population? To illustrate this concern, psychological researchers warn against oversampling from Western, Educated, Industrial, Rich, and Democratic (WEIRD) populations because they differ from their counterparts in important ways, including visual perception and spatial reasoning [6]. Examples of similar biases associated with user research can include: lack of prototypical cases; clustering effects; confounding factors; and value range restriction [7]. As such, it is important to facilitate broader collaboration through cross-cultural analyses and replications in different contexts. Such work can then be compared and combined (i.e., through meta-analyses) to form more generalizable findings.

As a first step, this paper proposes and evaluates the characteristics of a new video dataset to use as a standard benchmark: the CP-QAE-I. By sharing this video dataset, it is hoped that more nations become involved in this research. 


\section{RELATED WORK}

There has been much related work in the areas of perception of quality (e.g. [8], [9], [1]) and the experience of affect in multimedia (e.g. [10], [11]). Three key factors have been shown to influence these dependent variables, namely: human factors, the characteristics of the individual; system factors, the characteristics of the media, device and network used; and contextual factors, additional characteristics such as time, task, and local conditions [12].

It can be seen that empirical work in this area focuses on system factors with limited consideration for human factors. For example, there are studies which examine the influence of: (a) device characteristics [13]; (b) manipulating content, bit rate, frame rate and audio quality [2] (c) adaptation parameters such as playback time and switch amplitude in video streaming context [14], [15]; and (d) quality of service arbitration [16], [17]. However, even those that do take human factors into consideration and explore human-computer interaction typically only include basic demographic variables such as gender, age group and level of expertise [18], [19]. These may be insufficient to generalize the findings to a broader population because of the possibility of idiosyncratic rater effects [20].

Such rater effects may include emotional, physical and mental constitution because they each play a crucial role in the early sensory processing [21]. Additionally, previous experiences, socio-cultural expectations, goals, and values could influence evaluations of content by shaping what an individual considers to be 'good' or 'bad' quality. For example, there has been work which shows that personality influences media content preferences [22]. However, while there is work which explore these influences (e.g., [9], [23], [24], [25], [26]), many studies are based on subjective tests applied to samples drawn from specific populations. Consequently, it is important to determine if there is variation across different populations to permit broader generalizations. This underscores the importance of sharing datasets so such verification can be attempted.

\section{THE CP-QAE-I}

The CP-QAE-I (The Culture and Personality in Quality of Affect and Experience Dataset Version I) is a video dataset containing 144 video sequences in MP4 format. There is 1 nominal parameter: content. This is based on 12 short 'movie clips' which have been purposively selected to uniformly cover a wide range of affective categories from popular movies [27]. Movie clips of different valence, and which had least variation in ratings on arousal (by [27]), were taken for this study to minimize any content-based biases. The content parameter also varies in further important ways including the cinematographic techniques and the technologies that were used during the original production of the movies. Additionally, there are 3 ordinal parameters: bit-rate $(384 \mathrm{~kb} / \mathrm{s}$ and $768 \mathrm{~kb} / \mathrm{s})$; frame dimension (480p and 720p); and frame rate (5fps, $15 \mathrm{fps}$ and $25 \mathrm{fps})$. Thus, in the dataset, there are 12 quality conditions (resulting from the $3 * 2 * 2$ settings of frame-rate, resolution and bit-rate variables) and 12 emotion conditions (resulting from $2 * 6$ primary emotions [27]), thus resulting in a set of 144 'video sequences' $(12 * 12)$. Each video sequence has a length between 1 and 3 minutes.

\section{Evaluation Methodology}

\section{A. Procedure}

The video sequences were hosted on web server locally at each institution involved in the evaluation. Participants were asked to access an on-line questionnaire from the corresponding local network on which the web server was hosted (to avoid latency issues). They were informed that they would see several emotional video sequences and they would have to report their perceived quality and affect scores to the videos by filling out questionnaires after watching the clips. Consent and complete anonymity were assured at each stage.

Participants began by reporting demographic information. Then they were shown 14 video sequences: 2 training clips (one at the beginning of the survey and another during the middle as a reminder); and the 12 clips under assessment. The training clips showed participants what is considered 'high' quality. Each clip represents a different emotion condition so all participants were exposed to all emotion conditions. The order of these conditions was randomized, however these were drawn from two different sets of conditions (each randomly selected to be used before or after the middle training video) to ensure that two clips targeting the same emotion could not be shown consecutively. Additionally, participants were randomly allocated quality parameters for each individual video in the sequence (except for the training videos). The participants used $23^{\prime \prime}$ wide-screen monitors and a resolution of $1920 \times 1080$.

Participants were instructed to report what they had felt (in terms of quality, affect and enjoyment) and not what response they believed the content should induce in the viewers. They were also told that their assessment should be based on what they felt at the specific time watching the video, and not their general mood. As reported by Fredrickson and Kahneman [28], this procedure of retrospective evaluation of feeling when conducted according to instructions given by Philippot [29] is a good predictor of the actual state felt during the stimulation, and is particularly representative of the peak state felt by the end of the simulation.

\section{B. Measures}

1) Perceived Quality: To assess subjective perception of quality, the QoP-LoQ scale [30] was used. This consists of a single 5-point Likert-type item where participants indicate how they judged the quality of the video sequence. A score of 1 indicates "no" satisifcation while that of 5 indicates "absolute" satisfaction.

2) Positive and Negative Affect: To assess the affective response, the Differential Emotions Scale [31] was used. This includes 16 sets of of emotional adjectives. Each set was linked to a 5-point Likert-type item so participants could indicate the extent they felt the corresponding emotion. Aggregate scores for positive affect (i.e., joy, warth, love, calm, etc.) and negative affect (i.e., anger, fear, anxiety, sadness, etc.) were computed by a summation of the respective items.

\section{Participants}

A power analysis was conducted in $\mathrm{G}^{*}$ Power 3 to calculate the required sample size based on the use of the $F$-statistic and repeated measures. Using conventional error probabilities $(\alpha=$ 
TABle I. A FiXed-EfFect Multilevel Linear Regression Model Predicting Three Dependent Variables

\begin{tabular}{|c|c|c|c|c|c|c|c|c|c|c|}
\hline \multirow[b]{2}{*}{ Parameter } & \multirow[b]{2}{*}{$d f_{n u m}$} & \multicolumn{3}{|c|}{ Perceived Quality } & \multicolumn{3}{|c|}{ Positive Affect } & \multicolumn{3}{|c|}{ Negative Affect } \\
\hline & & $d f_{d e n}$ & $F$ & $p$ & $d f_{d e n}$ & $F$ & $p$ & $d f_{d e n}$ & $F$ & $p$ \\
\hline Movie Clip & 11 & 139.11 & 7.423 & .000 & 156.009 & 25.315 & .000 & 144.643 & 33.932 & .000 \\
\hline Frame Rate & 2 & 873.31 & 13.324 & .000 & 803.739 & .321 & .725 & 710.192 & .056 & .946 \\
\hline Frame Dimension & 1 & 885.98 & 14.735 & .000 & 809.889 & .006 & .939 & 729.398 & 3.298 & .070 \\
\hline Bit-Rate & 1 & 880.69 & 14.431 & .000 & 816.675 & 1.724 & .190 & 714.909 & .304 & .582 \\
\hline Frame Rate $*$ Bit-Rate & 2 & 875.53 & 4.546 & .011 & 807.481 & .127 & .881 & 716.290 & .296 & .744 \\
\hline Frame Dimension $*$ Bit-rate & 1 & 882.80 & 7.023 & .008 & 813.320 & .694 & .405 & 718.919 & 1.039 & .308 \\
\hline Frame Rate $*$ Frame Dimension $*$ Bit-Rate & 2 & 874.86 & 5.888 & .003 & 804.925 & .128 & .880 & 717.356 & .871 & .419 \\
\hline Nationality & 3 & 884.24 & 5.378 & .001 & 804.070 & 4.164 & .006 & 722.020 & 16.389 & .001 \\
\hline
\end{tabular}

$.05, \beta=0.2)$ and assuming medium effects $(f=0.39)$ with correlation $(r=0.8)$, a minimum size of 64 was suggested. The participants were 76 university students drawn from the computing departments of the authors' institutions. These were 41 British, 12 Chinese, 14 Indian, and 11 Singaporean students; all natives. The proportion of female participants was $25.6 \%$ and the mean age was 23 years $(\sigma=3.74)$.

\section{RESUlTS \& DisCUSSION}

Analyses were conducted in PASW 18.0.3 for Windows. To determine whether sufficient distinctiveness between the video sequences could be found in the dataset, three fixed-effect multilevel linear regression models were defined (i.e., one for each dependent variable) [32]. A diagonal residual covariance matrix was used to account for repeated measures. The parameters in each model were estimated together using the restricted maximum-likelihood method. No data were missing, however unbalanced cells should be noted as a limitation. Prior to the analysis, data were standardized into z-scores (a value of 0 represents the population mean and each unit represents a standard deviation). This has been done to facilitate comparisons in terms of within-variable variation.

Table I shows the three regression models with each predicting a different dependent variable. These dependent variables are: perceived quality; positive affect; and negative affect. Several independent variables are within-subject parameters. These are: movie clip; frame rate; frame dimension; and bit-rate. The independent variable nationality has also been included, but as a between-subject parameter. Also shown are the (numerator and denominator) degrees of freedom $(d f)$, the $F$-statistic, and their associated $p$-value. These are used to determine whether there is sufficient evidence to conclude whether a parameter is different from zero (i.e., the null hypothesis is rejected). This is known as statistical significance and is determined at the conventional level $(p<.05)$ using two-tailed tests.

\section{A. Perceived Quality}

The first group in Table I shows the analysis with perceived quality as the dependent variable. This reveals that most of the parameters are significant. It should be noted that in addition to the system parameters, the movie clip itself is a significant parameter. This illustrates that the nature of content influences the perception of its quality. Figures 1 and 4 show the relative extent of this in terms of mean $\mathrm{z}$-value differences between each movie clip and system parameter setting, respectively.
It is also interesting to note that content which evokes strong positive affects (e.g., FOREST_GUMP) tended to be perceived as higher quality $(r=.252, p<.000)$. Parameter interactions are also present. Thus, the mean perceived quality of clips with "higher" settings are sometimes lower than expected (see C-I in Table II - 25fps, 720p, 768kbps score is lower than that at $5 \mathrm{fps}, 480 \mathrm{p}$ and $384 \mathrm{kbps})$. This supports prior findings about complexity in quality of perception assessment [1]. It would be interesting to see if this property varies across cultures and personalities.

\section{B. Positive Affect}

The second group in Table I shows the results of the regression analysis with positive affect as dependent variable. As anticipated, the movie clip was significant. Some system predictors such as \{Frame Rate $*$ Frame Dimension\} also approached significance $(p=.06)$. It should be noted, however, that Figure 2 shows positive affect was not uniform across the movie clips with two clips (FOREST_GUMP and DEAD_POETS_SOCIETY_2) having very high positive affect compared to the other movie clips. Figure 5 shows that the ranges of the mean z-values are small (less than 1 standard deviation) while the confidence intervals overlap. This suggests that 'higher' parameter settings do not necessarily lead to participants experiencing more positive affects.

\section{Negative Affect}

The third and final group in Table I shows the results of the regression analysis with negative affect as dependent variable. As expected, the movie clip is significant and predicts the most variance. This is shown more clearly in Figure 3 where the mean $\mathrm{z}$-values vary across each movie clip without skew. No system parameter was a significant predictor. This can be seen in Figure 6 which, although shows large confidence intervals, illustrates that 'lower' parameter settings do not necessarily lead to stronger negative affect (i.e., frustration with low quality).

\section{Nationality}

The analyses in Table I show that nationality is consistently statistically significant in each of the three models, suggesting cultural influence. This supports the rationale for further research using the dataset. Table IV reveals differences in mean z-values between the nations. These differences can be seen clearly for perceived quality and negative affect, with the difference in positive affect being smaller in comparison. 


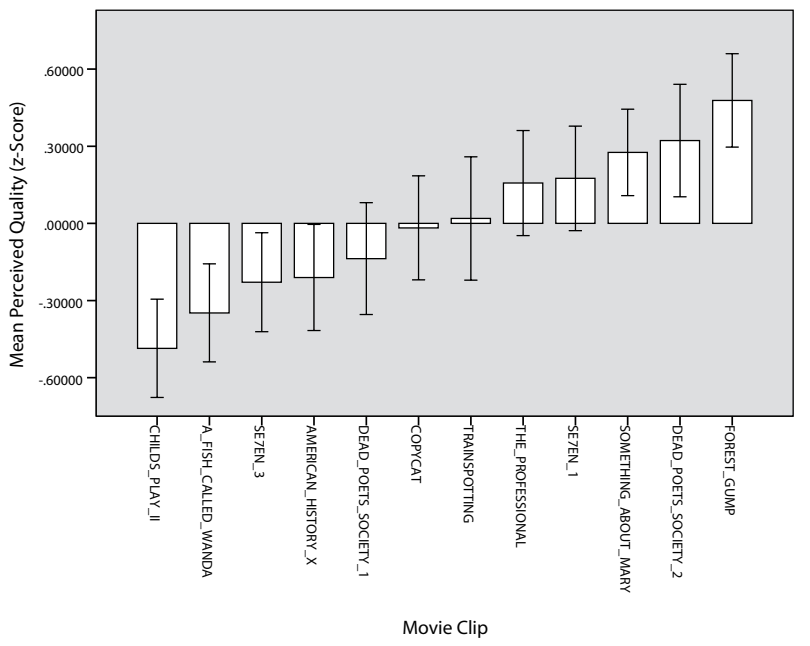

Fig. 1. Relative mean levels of perceived quality that participants rated each video sequence.

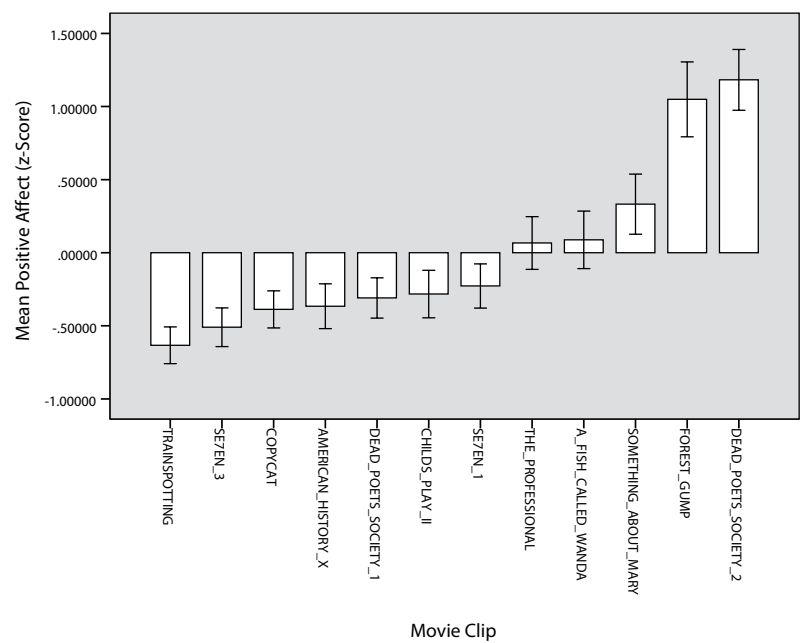

Fig. 2. Relative mean levels of positive affect that participants rated each video sequence.

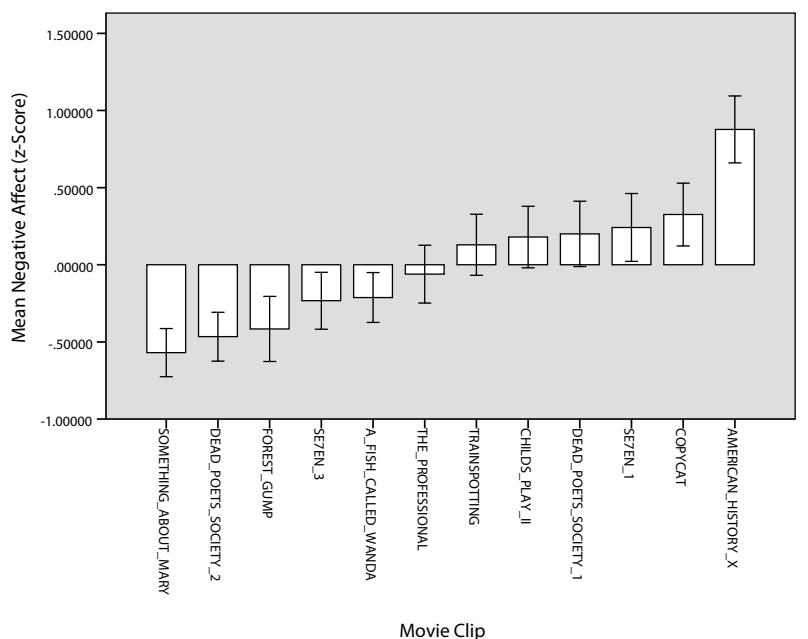

Fig. 3. Relative mean levels of negative affext that participants rated each video sequence.

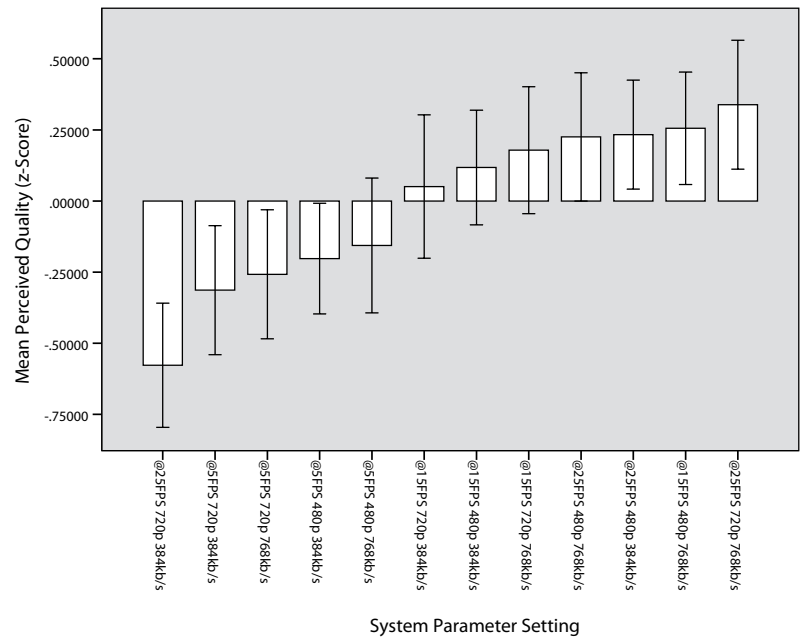

Fig. 4. Relative mean levels of perceived quality that participants rated each quality condition.

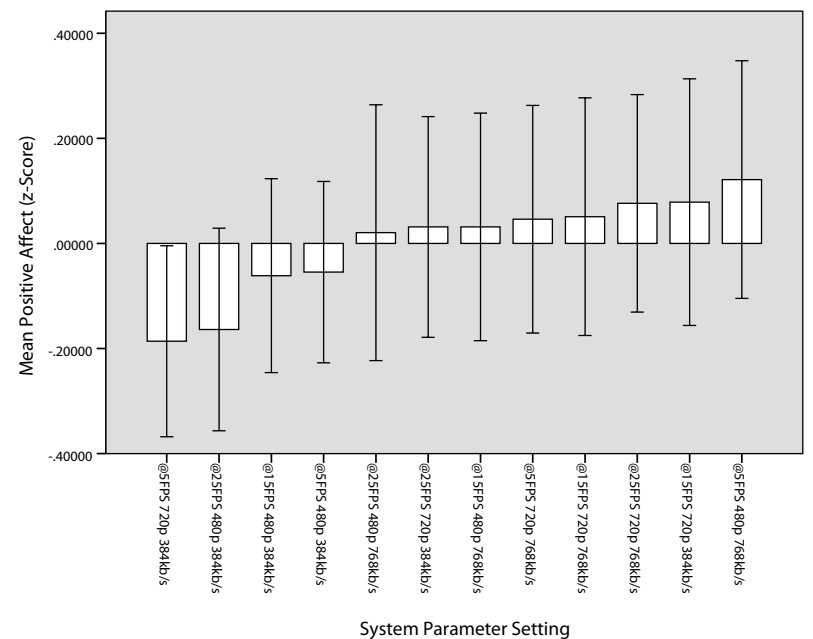

Fig. 5. Relative mean levels of positive affect that participants rated each quality condition.

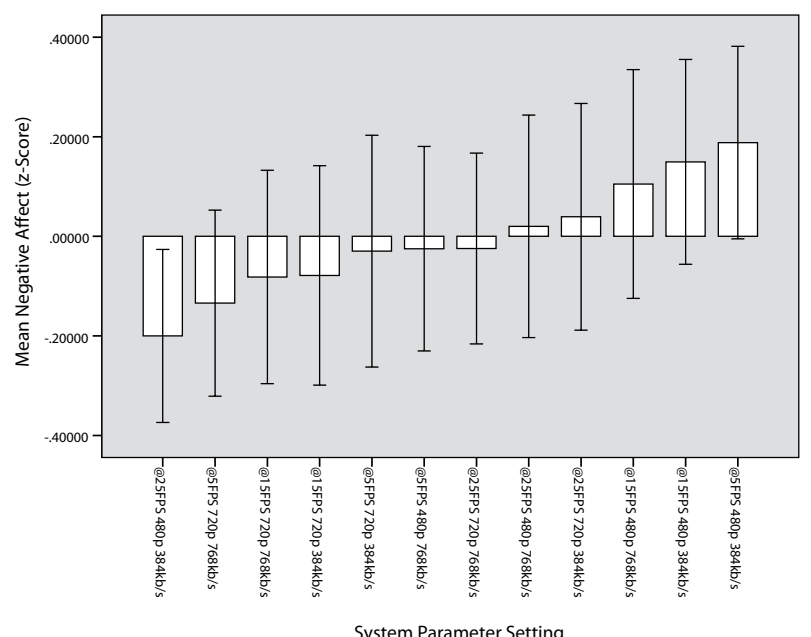

Fig. 6. Relative mean levels of negative affect that participants rated each quality condition. 

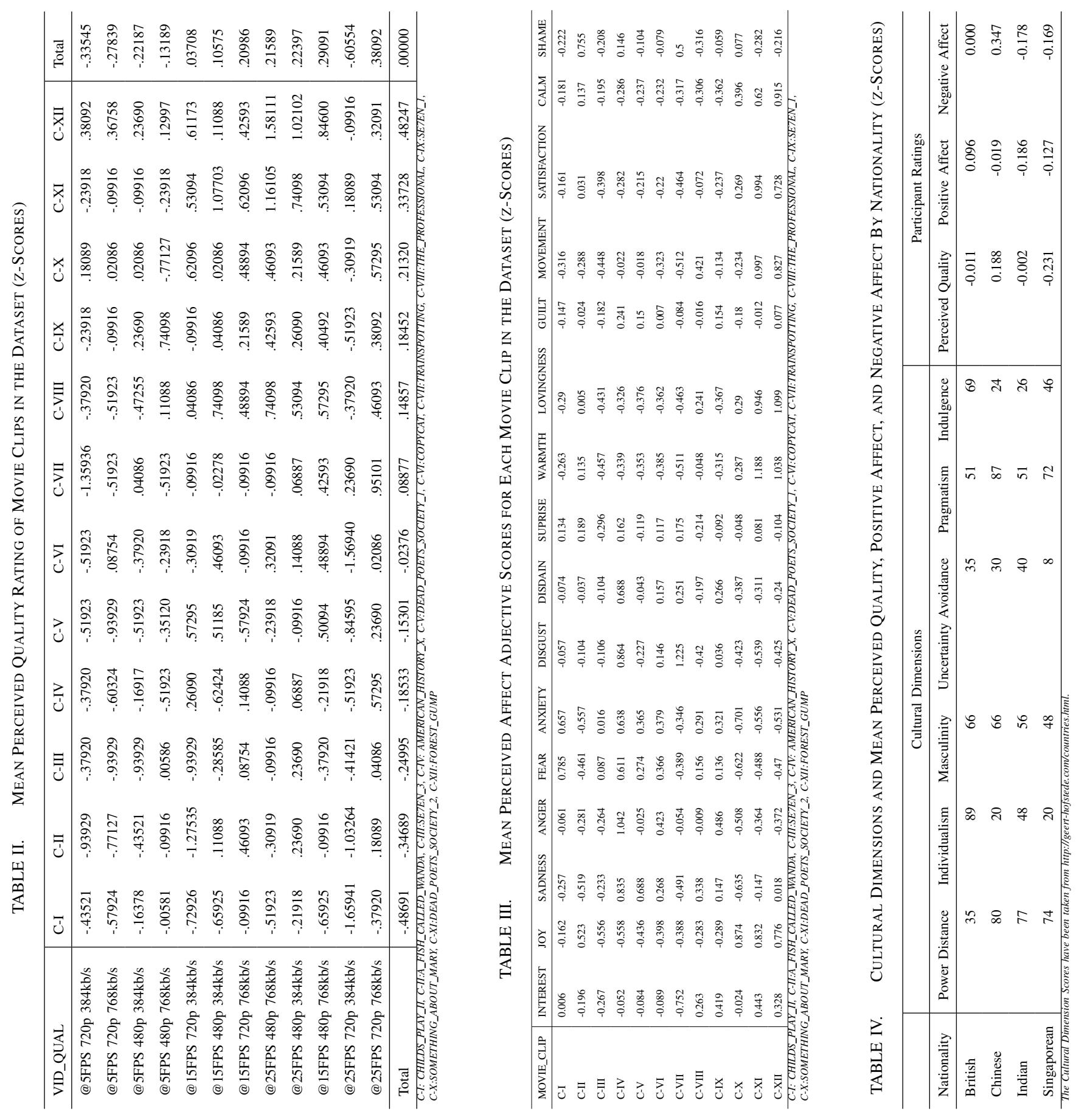


\section{CONCLUSION}

This paper presents the CP-QAE-I, a video dataset containing 144 video sequences based on 12 short movie clips. An analysis of its characteristics shows that the video sequences it contains are distinct and vary considerably in terms of perceived quality as well as positive and negative affect. Of particular note is the finding that the nationality is a significant predictor of ratings for all three dependent variables, suggesting that national culture may interact with the way in which participants rate video sequences. As such, use of the dataset to explore the influence of human factors such as personality and culture on perceived quality and the experience of affect is encouraged. This will support broader research into the interactions between human and system factors. In addition, the results can then be integrated to help (re-)discover generalizable findings about human psychology from the perspective of multimedia perception, while also supporting the development of novel personalized multimedia content delivery systems.

\section{ACKNOWLEDGEMENT}

A part of this work was carried out at the Rapid-Rich Object Search (ROSE) Lab at the Nanyang Technological University, Singapore. The ROSE Lab is supported by a grant from the Singapore National Research Foundation. This grant is administered by the Interactive \& Digital Media Programme Office at the Media Development Authority, Singapore.

\section{AVAILABILITY}

The dataset can be downloaded from: 1drv.ms/1M1bnwU

\section{REFERENCES}

[1] G. Ghinea and J. P. Thomas, "Qos impact on user perception and understanding of multimedia video clips," in Proceedings of the sixth ACM international conference on Multimedia. ACM, 1998, pp. 49-54.

[2] A. Oeldorf-Hirsch, J. Donner, and E. Cutrell, "How bad is good enough?: exploring mobile video quality trade-offs for bandwidth-constrained consumers," in Proceedings of the 7th Nordic Conference on Human-Computer Interaction. ACM, 2012, pp. 49-58.

[3] G. Matthews, I. J. Deary, and M. C. Whiteman, Personality traits. Cambridge University Press, 2003.

[4] L. R. Goldberg, "An alternative" description of personality": the big-five factor structure." Journal of personality and social psychology, vol. 59, no. 6, p. $1216,1990$.

[5] G. Hoftede, G. J. Hofstede, and M. Minkov, Cultures and organizations: software of the mind: intercultural cooperation and its importance for survival. McGraw-Hill, 2010.

[6] J. Henrich, S. J. Heine, and A. Norenzayan, "The weirdest people in the world?" Behav. \& Brain Sci., vol. 33, no. 2, pp. 61-83, 2010.

[7] R. N. Landers and T. S. Behrend, "An inconvenient truth: Arbitrary distinctions between organizational, mechanical turk, and other convenience samples," Industrial and Organizational Psychology, pp. $1-38$, in press.

[8] M. Fiedler, T. Hossfeld, and P. Tran-Gia, "A generic quantitative relationship between quality of experience and quality of service," Network, IEEE, vol. 24, no. 2, pp. 36-41, 2010.

[9] B. Rainer, M. Waltl, E. Cheng, M. Shujau, C. Timmerer, S. Davis, I. Burnett, C. Ritz, and H. Hellwagner, "Investigating the impact of sensory effects on the quality of experience and emotional response in web videos," in QoMEX. IEEE, 2012, pp. 278-283.

[10] X.-P. Gao, J. H. Xin, T. Sato, A. Hansuebsai, M. Scalzo, K. Kajiwara, S.-S. Guan, J. Valldeperas, M. J. Lis, and M. Billger, "Analysis of cross-cultural color emotion," Color Research \& Application, vol. 32, no. 3, pp. 223-229, 2007.
[11] B. Manav, "Color-emotion associations and color preferences: A case study for residences," Color Research \& Application, vol. 32, no. 2, pp. 144-150, 2007.

[12] U. Reiter, K. Brunnström, K. De Moor, M.-C. Larabi, M. Pereira, A. Pinheiro, J. You, and A. Zgank, "Factors influencing quality of experience," in Quality of Experience. Springer, 2014, pp. 55-72.

[13] G. Ghinea and K. J. Patterson, "Perceived multimedia quality: The impact of device characteristics," in HCI International 2011-Posters Extended Abstracts. Springer, 2011, pp. 143-146.

[14] T. Hoßfeld, M. Seufert, T. Zinner, and C. Sieber, "Crowdsourced subjective user study results on qoe influence factors of http adaptive streaming," Tech. Rep. 491, University of Würzburg, Tech. Rep., 2014.

[15] M. Seufert, S. Egger, M. Slanina, T. Zinner, T. Hoßfeld, and P. Tran-Gia, "A survey on quality of experience of http adaptive streaming."

[16] M. Siller and J. Woods, "Improving quality of experience for multimedia services by qos arbitration on a qoe framework," in in Proc. of the 13th Packed Video Workshop 2003. Citeseer, 2003.

[17] G. Ghinea and J. Thomas, "An approach towards mapping quality of perception to quality of service in multimedia communications," in Multimedia Signal Processing, 1999 IEEE 3rd Workshop on. IEEE, 1999, pp. 497-501.

[18] D. Geerts, K. De Moor, I. Ketyko, A. Jacobs, J. Van den Bergh, W. Joseph, L. Martens, and L. De Marez, "Linking an integrated framework with appropriate methods for measuring qoe," in QoMEX. IEEE, 2010, pp. 158-163.

[19] M. Quintero and A. Raake, "Towards assigning value to multimedia qoe," in QoMEX, Sept 2011, pp. 1-6.

[20] S. E. Scullen, M. K. Mount, and M. Goff, "Understanding the latent structure of job performance ratings." Journal of Applied Psychology, vol. 85 , no. 6, p. $956,2000$.

[21] E. Goldstein, Sensation and perception. Cengage Learning, 2013.

[22] S. C. Guntuku, S. Roy, and W. Lin, "Personality modeling based image recommendation," in MultiMedia Modeling. Springer, 2015, pp. 171-182.

[23] G. Ghinea and S. Y. Chen, "The impact of cognitive styles on perceptual distributed multimedia quality," British Journal of Educational Technology, vol. 34, no. 4, pp. 393-406, 2003.

[24] M. Hyder, N. Crespi, M. Haun, C. Hoene et al., "Are qoe requirements for multimedia services different for men and women? analysis of gender differences in forming qoe in virtual acoustic environments," in Emerging Trends and Applications in Information Communication Technologies. Springer, 2012, pp. 200-209.

[25] J. A. Redi, Y. Zhu, H. de Ridder, and I. Heynderickx, "How passive image viewers became active multimedia users," in Visual Signal Quality Assessment. Springer, 2015, pp. 31-72.

[26] E. Siahaan, J. Redi, and A. Hanjalic, "Beauty is in the scale of the beholder: Comparison of methodologies for the subjective assessment of image aesthetic appeal," in QoMEX, Sept 2014, pp. 245-250.

[27] A. Schaefer, F. Nils, X. Sanchez, and P. Philippot, "Assessing the effectiveness of a large database of emotion-eliciting films: A new tool for emotion researchers," Cognition and Emotion, vol. 24, no. 7, pp. 1153-1172, 2010.

[28] B. L. Fredrickson and D. Kahneman, "Duration neglect in retrospective evaluations of affective episodes." Journal of personality and social psychology, vol. 65, no. 1, p. 45, 1993.

[29] P. Philippot, "Inducing and assessing differentiated emotion-feeling states in the laboratory," Cognition \& Emotion, vol. 7, no. 2, pp. 171-193, 1993.

[30] S. R. Gulliver and G. Ghinea, "Defining user perception of distributed multimedia quality," ACM Transactions on Multimedia Computing, Communications, and Applications, vol. 2, no. 4, pp. 241-257, 2006.

[31] G. J. McHugo, C. A. Smith, and J. T. Lanzetta, "The structure of self-reports of emotional responses to film segments," Motivation and Emotion, vol. 6, no. 4, pp. 365-385, 1982.

[32] R. Bosker and T. Snijders, "Multilevel analysis: An introduction to basic and advanced multilevel modeling, 2nd ed," New York, 2012. 